\title{
FREE-FREE EMISSION AND THE BIG BLUE BUMP
}

\author{
RICHARD BARVAINIS \\ MIT/Haystack Observatory, Westford MA 01886 USA
}

\begin{abstract}
.
Optically thin thermal emission may be a viable alternative to optically thick accretion disks for the origin of the optical/UV Big Blue Bump. In this contribution I summarize a number of points favoring the optically thin, or free-free, hypothesis. The free-free model is consistent with the most stringent quasar size and luminosity constraints provided sources are composed of a large number of very small and dense cloudlets or filaments.
\end{abstract}

The most luminous continuum feature in quasars is the optical/UV Big Blue Bump (BBB). The origin of the BBB has been ascribed to thermal emission from geometrically thin, optically thick accretion disks, or to synchrotron from the vicinity of a supermassive black hole. It has also been suggested more recently that the BBB might be optically thin thermal emission, i.e. predominantly free-free.

The poster outlined here summarized observational evidence favoring the freefree mechanism, and briefly discussed some theoretical constraints on the nature of the sources. Detailed arguments and references can be found in Barvainis (1993, ApJ 412, 513). Some points in favor of the free-free hypothesis are:

1) Optical/UV slope matches mean of quasars $\left(<\alpha_{\mathrm{opt}}>\approx-0.3\right)$

2) Compatible with thermal dust emission in IR.

3) Predicts low polarization.

4) $T_{B}$ can be high $\left(>10^{5} \mathrm{~K}\right)$.

5) Optically thin continuum in Mrk 509 inferred from ultrabroad wings.

6) Optical/UV variations predicted to be broadband, simultaneous.

7) Reprocessed X-rays can be consistent with optically-thin opt/UV.

8) Lyman edge very weak for hot sources $\left(T \sim 10^{6} \mathrm{~K}\right)$.

Some theoretical constraints: Source temperatures must be in the range $10^{5}-$ few $\times 10^{6} \mathrm{~K}$ in order to satisfy both the optical/UV spectral shape and the soft X-ray excess.

In order to satisfy quasar size/luminosity constraints (in particular a $\mu$ lensing constraint on Q2237+0305), sources must be composed of a large number of very small and dense clouds $\left(r \sim 10^{7} \mathrm{~cm}, n \sim 10^{15} \mathrm{~cm}^{-3}\right)$. Past critics of the free-free model have considered single uniform sources, which certainly do not work.

For free-free sources with $T \sim 10^{6} \mathrm{~K}$, the Lyman edge is intrinsically weak. There is evidence from UV slopes that high luminosity sources have high temperatures. The Lyman edge has been searched for only in moderate to high $L$ sources, where $T$ may be high and the Lyman edge intrinsically weak.

T. J.-L. Courvoisier and A. Blecha: Multi-Wavelength Continuum Emission of AGN, 344.

C 1994 IAU. Printed in the Netherlands. 